\title{
Principales aspectos metodológicos en el estudio del estrés laboral en personal universitario: Una revisión sistemática
}

\author{
José Luis Rojas-Solís \\ Benemérita Universidad Autónoma de Puebla, Puebla, México \\ https://orcid.org/0000-0001-6339-4607 \\ Guadalupe Flores-Meza \\ Benemérita Universidad Autónoma de Puebla, Puebla, México \\ https://orcid.org/0000-0002-3257-2498 \\ Irma Guadalupe Cuaya-Itzcoatl \\ Benemérita Universidad Autónoma de Puebla, Puebla, México \\ https://orcid.org/0000-0003-0665-071X
}

Recibido:09/08/20 Revisado: 10/09/20 Aceptado: 01/11/20 Publicado: 13/11/20

\begin{abstract}
Resumen
Introducción: El estudio del estrés laboral en el ámbito universitario no ha estado exento de dificultades conceptuales y metodológicas. Objetivo: analizar los principales aspectos metodológicos e instrumentales en estudios publicados entre 2010 y 2019, sobre estrés laboral en docentes, administrativos y personal de apoyo que trabaja en universidades latinoamericanas. Método: siguiendo lo sugerido por el modelo PRISMA, se consultaron las bases de datos: La Referencia, Dialnet, Scopus, EBSCO y Web of Science, obteniendo inicialmente 258 trabajos que dieron lugar a 6 estudios para su revisión final. Resultados: señalan que los estudios fueron cuantitativos, no experimentales y transversales, y con alcances descriptivos. Además, se detectó el uso de 15 instrumentos que principalmente evalúan estrés laboral y factores de riesgo laborales. Discusión: se resaltan niveles significativos de estrés laboral en las muestras incluidas, así como la necesidad de prevenir las causas y factores asociados al fenómeno.
\end{abstract}

Palabras clave: Estrés; Estrés laboral; Personal universitario; Revisión sistemática.

\section{Main methodological issues in the study of job stress in college staff: A systematic review}

\begin{abstract}
Introduction: Research about of job stress at university contexts has not been exempt from conceptual and methodological difficulties. Objective: analyze the main methodological and instrumental aspects in studies published between 2010 and 2019, on job stress in professor, administrative and support staff working in Latin American universities. Method: following the suggested by the PRISMA model, it was consulted the databases: LA Reference, Dialnet, Scopus, EBSCO and Web of Science, obtaining 258 papers that, after a debugging process, derivated 6 studies for their final review. Results: the results show that all the studies were quantitative, with non-experimental and cross-sectional designs, and with descriptive scopes. In addition, the use of 15 instruments that mainly assess work stress and occupational risk factors was detected. Theoretical and methodological implications derived from the findings are discussed.
\end{abstract}

Key words: stress; job stress; college staff; systematic review. 


\title{
Principais aspectos metodológicos no estudo do stresse ocupacional no pessoal universitário: Uma revisão sistemática
}

\begin{abstract}
Resumo
As pesquisas sobre estresse no trabalho em contextos universitários não ficaram isentos de dificuldades conceituais e metodológicas, portanto o objetivo desta revisão sistemática é analisar os principais aspectos metodológicos e instrumentais em estudos publicados entre 2010 e 2019, sobre estresse no trabalho em professores, administrativos e pessoal de apoio que trabalha em universidades públicas latino-americanas. Seguindo o sugerido pelo modelo PRISMA, foram consultadas as bases de dados: LA Reference, Dialnet, Scopus, EBSCO e Web of Science, obtendo 258 artigos que, após um processo de depuração, obtiveram 6 estudos para sua revisão final. Os resultados mostram que todos os estudos foram quantitativos, com delineamento não experimental e transversal, e com escopo descritivo. Além disso, foi detectada a utilização de 15 instrumentos que avaliam principalmente o estresse no trabalho e os fatores de risco ocupacionais. As implicações teóricas e metodológicas derivadas dos resultados são discutidas.
\end{abstract}

Palavras-chave: estresse; estresse no trabalho; equipe da faculdade; revisão sistemática.

Citar como:

Rojas-Solís, J., Flores-Meza, G. \& Cuaya-Itzcoatl, I. (2021). Principales aspectos metodológicos en el estudio del estrés laboral en personal universitario: Una revisión sistemática. Revista Digital de Investigación en Docencia Universitaria. 15(1), e1248. https://doi.org/ 10.19083/ridu.2021.1248

$\mathrm{L}$ a situación laboral actual en la Universidad presenta numerosos desafíos al personal universitario como docentes, administrativos y personal de apoyo, así se han realizado diversas investigaciones para intentar delimitar y entender fenómenos indeseables como el estrés laboral. En ese contexto la presente revisión sistemática exploratoria se encamina a analizar los principales aspectos metodológicos e instrumentales en estudios sobre estrés en personal que labora en universidades públicas, divulgados entre los años 2010 y 2019. Para ello se han consultado cinco bases de datos de reconocido prestigio con el objetivo ulterior de analizar la evidencia científica acumulada y hacer disponible la síntesis obtenida para consulta y uso de quien esté interesado en la materia.

\section{Introducción}

Considerado como la enfermedad del siglo XXI, el estrés ha sido objeto de interés en la investigación debido a los efectos que genera en la salud de las personas y en su ambiente de trabajo, por tanto se le considera un padecimiento que repercute en la calidad de vida (Bairero, 2017) y afecta sin distinción de características personales (Karam et al., 2019). El origen de este fenómeno puede ubicarse en problemas domésticos o laborales (Organización Mundial de la Salud [OMS], 2004) siendo una categoría derivada el estrés laboral (Berrío \& Mazo, 2011), el cual en las organizaciones genera y representa altos costos por la gran incidencia de empleados que lo padecen (García et al., 2013). Es así que, debido a esta afección, la salud laboral tiende a declinar por factores como, por ejemplo, la estancia en el trabajo (Collado et al., 2016). En ese sentido, las personas pueden experimentar estrés laboral cuando las exigencias del trabajo exceden sus recursos mentales y físicos, las cuales pueden ser o no considerados amenazantes o dañinas, dependiendo la capacidad del individuo (Hessels et al., 2017; Meurs \& Perrewé, 2011).

Por otro lado, cabe resaltar el creciente interés en investigar el estrés laboral en países en desarrollo (Ramírez, 2019), sin olvidar la existencia de dilemas teóricos, conceptuales y metodológicos en su estudio, por lo que este trabajo realiza una revisión sistemática de la literatura con el fin de 
Rojas-Solís, J., Flores-Meza, G. \& Cuaya-Itzcoatl, I.

obtener un panorama actual sobre la materia enfocándose en aspectos metodológicos e instrumentales.

\section{Estrés laboral y el contexto universitario}

Los cambios vividos por la humanidad han modificado la forma de producir la vida, la cultura y la sociedad, marcando así una época de transformaciones en las personas (Mejía, 2015). En ese orden de ideas, es conveniente recordar que el trabajo es el medio por el cual se obtiene el sustento económico y se suplen necesidades psicológicas como la autorrealización personal y profesional, la construcción de la identidad social o el sentido de vida en comunidad (Alves et al., 2013). Sin embargo es ahí también donde, paradójicamente, la gente podría preferir trabajar en malas condiciones que estar desempleada (Organización Internacional del Trabajo [OIT], 2016). Es en este entorno actual que existe un incremento en las demandas del desempeño del trabajador, debiendo aumentar su capacidad de flexibilidad, trabajo en grupo, toma de decisiones y dominio de las nuevas tecnologías de la información y las comunicaciones (Ramos \& Jordão, 2015), es así como se presenta una interacción entre demandas percibidas en la situación, los recursos que el trabajador tiene para amortiguar el estresor y las características individuales del mismo (Hermosa \& Perilla, 2015).

En ese sentido, el estrés se manifiesta en diversos ámbitos, dos de los más frecuentes son la vida cotidiana y el trabajo, no obstante, pese a su carácter situacional, sus efectos parecen no desaparecer de inmediato al cambiar los individuos de contexto (Nava et al., 2016). En cuanto al estrés laboral, éste surge como una reacción ante las exigencias y demandas laborales (Patlán, 2019), las cuales aunadas a la ausencia de control para tomar decisiones en el trabajo pueden causar tensión en el personal (Birolim et al., 2019; Karasek, 1979), originando así una respuesta emocional, fisiológica y conductual potencialmente patógena que influye en la actividad del trabajador y en el resultado de sus actividades (Zuniga-Jara \& Pizarro-Leon, 2018). Sin embargo, es conveniente señalar que este proceso puede ser diferente en cada individuo al adoptarse una postura de afrontamiento re- lacionada con emociones positivas, cognición y aprendizajes que se hayan obtenido como fruto de experiencias ante el estrés (Ganster \& Rosen, 2013; McCarthy, 2019; Meurs \& Perrewé, 2011).

En el actual mundo globalizado, la universidad es el ente que contribuye a impulsar el desarrollo económico y social de un país (Huanco, 2019). Se trata de un espacio organizado que proporciona sentido de representación, significación y ordenamiento de comportamientos, rituales y roles para grupos relacionados a ella, así las universidades representan valores, creencias y expectativas de diversos estratos, grupos y clases sociales (Acosta, 2019); y su desafío es contribuir a la sociedad, en la formación de un profesional con conocimiento en materia, pero principalmente en competencias y valores (Figueroa et al., 2019).

Por ello, sus integrantes comparten fines para lograr objetivos en común, manteniendo como base un trabajo colaborativo encaminado a una cultura laboral (Aparicio \& Sepúlveda, 2019). Sin embargo, a partir de los años sesenta, la globalización marcó cambios en las organizaciones, como las universidades de América Latina y el Caribe, desvaneciendo las humanidades y haciendo primordial lo tecnológico (Soto \& Forero, 2016). Por su parte, el contexto globalizador ha favorecido reformas y políticas educativas con la visión de elevar la calidad de la educación, así a partir de estas modificaciones las universidades públicas se han visto precisadas a adoptar un sistema de control de calidad que les permita lograr lo tan anhelado (Zúñiga et al., 2016). Sin embargo, ante los cambios mundiales de transición entre el siglo XX y el XXI, algunos sistemas educativos han quedado obsoletos traduciendo las mínimas oportunidades educativas y la baja calidad en educación, en falta de oportunidades laborales, delincuencia y deterioro de la calidad de vida (Orozco-Alvarado et al., 2019). Así, ante las transiciones globales, las principales figuras que hacen frente y han estado a la par de los constantes cambios son las personas inmersas en el contexto universitario. En ese tenor, es importante señalar que docentes, personal administrativo y de apoyo son algunos sectores que forman la universidad $y$, por ende, se han visto afectados por los constantes cambios. 


\section{Docentes universitarios}

Se consideran agentes de cambio que poseen compromiso para afrontar, mediante la educación, los retos que se planteen poniendo en marcha las estrategias pedagógicas para que el estudiantado adquiera conocimientos ambientales y desarrolle un pensamiento crítico, sistémico y complejo de la realidad (Sánchez-Contreras \& Murga-Menoyo, 2019). Sin embargo, su exigencia laboral va en aumento pues se esperan mejores resultados en el menor tiempo posible, generando en ellos baja autoestima y sensación de fracaso (González \& Orta, 2016); sumado a ello, el tipo de universidad podría ser un factor que influye en la diferencia de niveles de carga de trabajo y de funciones en dicha población (Yousefi \& Kanesan, 2019). Es así como el docente al encontrarse en un mundo lleno de constantes transformaciones, como el actual, debe realizar sus actividades con urgencia y presión lo que en el transcurso del tiempo le puede ciertamente generar estrés (Alvites-Huamaní, 2019), derivando en estrés laboral, un tipo de estrés que se origina por el desequilibrio de las exigencias y presiones que se enfrentan por la coerción en el trabajo y las condiciones laborales no óptimas, excediendo la resistencia y capacidad del trabajador para poder enfrentarlas $\mathrm{y}$ resolverlas (Sánchez, 2017), afectando así su competencia personal y profesional sin olvidar su productividad (Watts \& Robertson, 2011). Al respecto, es preciso recordar que la docencia es una de las actividades más nobles del quehacer humano, caracterizada por poseer un amor a la sabiduría y a los educandos (Barrientos, 2016), empero también ha sido una de las profesiones asistenciales que se ve afectada, frecuentemente, por el estrés laboral (Botero, 2012).

\section{Administrativos}

El personal administrativo es considerado el talento humano encargado de desarrollar los procesos administrativos, académicos y presupuestales de la universidad, y funge como contacto de usuarios internos y externos, por ende, su labor está marcada por exigencias y constante presión debido a que debe ir a la par del desarrollo activo y productivo del mundo de hoy (García et al., 2014; Gonzáles, 2014). Así, esta población se considera vulnerable al encontrarse en constante tensión y en riesgo de sufrir estrés laboral, ya que está dentro de escenarios con los que debe lidiar usualmente, por ejemplo, con los tiempos límites de entrega, los documentos que deben realizar y la carga laboral, entre otros (Rivera-Encinas, 2016).

\section{Personal de apoyo}

Otro sector que forma la universidad es el personal de apoyo, el cual se integra por cuerpos no académicos y permanentes (González \& Codagnone, 2010). Se trata de un grupo que podría gozar de puestos estables y beneficios económicos en tiempos de crisis laboral y que, a diferencia de los sectores anteriores, no se hallaría expuesto a la competitividad del mundo profesional (Marsollier \& Aparicio, 2014). Añadido a ello, podría ameritar una subclasificación por la índole de sus diferentes tareas (administrativas, técnicas, profesionales, de mantenimiento y de servicios) por lo que es factible encontrar aportaciones y demandas diversas en estos subgrupos (Durán et al., 2010) y, por supuesto, estrés laboral.

Por otro lado, es pertinente mencionar la existencia de algunos estudios en la región iberoamericana y que comparten algunas características directas con la presente revisión sistemática en el contexto universitario, entre ellos se encuentran algunas tesis (García, 2016; Ramírez, 2018) o artículos empíricos (Carpio et al., 2017; Londoño, 2019). En cuanto a antecedentes de revisiones se puede mencionar al aporte de Avargues y Borda (2010), en el cual se hace una revisión de las principales líneas de investigación sobre el estrés laboral y burnout en la universidad, teniendo como muestra al personal docente, investigador, de administración y servicios. Por lo tanto, se hace evidente la falta de revisiones sistemáticas recientes sobre el estrés laboral y, en especial, con un enfoque en la región de América Latina. Es así como en el presente estudio se elaboraron las siguientes preguntas de investigación: ¿Cuáles son los principales aspectos metodológicos en el estudio del estrés laboral en el personal universitario? Y ¿qué instrumentos se han utilizado para ello? En ese sentido el objetivo es analizar los principales aspectos metodológicos en estudios publicados entre los años 2010 y 2019 sobre estrés laboral en docentes, administrativos y personal de apoyo que trabajan en universidades 
Rojas-Solís, J., Flores-Meza, G. \& Cuaya-Itzcoatl, I.

públicas; así como identificar los instrumentos utilizados para medir y/o evaluar el estrés laboral en personal universitario.

\section{Metodología}

Se trata de un diseño de investigación observacional y retrospectivo con el objetivo de sintetizar los resultados de investigaciones primarias (Beltrán, 2005), para llevar a cabo esta revisión sistemática exploratoria se siguió lo sugerido por el modelo PRISMA (Moher et al., 2009) en la mayor medida de lo posible, ello debido a la gran heterogeneidad de la naturaleza y diseño de los estudios evaluados.

Así se realizaron combinaciones de las palabras clave seleccionadas con el uso del operador booleano "AND", a partir de esto se obtuvieron dos cadenas de búsqueda, quedando en español: "Estrés laboral AND Personal AND Universitario" y en inglés: "Job stress AND College staff". La búsqueda de artículos se realizó en las bases de datos LA Referencia, Dialnet, Scopus, EBSCO y Web of Science, en el periodo de mayo a agosto de 2019, y se obtuvieron a partir de los siguientes:

Criterios de inclusión: 1) publicaciones realizadas en el periodo de tiempo de enero de 2010 a julio de 2019; 2) idioma inglés y español; 3) acceso abierto y texto completo; 4) estrés laboral como tema principal; 5) muestra de docentes, administrativos y personal de apoyo que labore en universidades de América Latina; 6) tener al menos un instrumento validado, que evalúe o mida estrés laboral para población de América Latina.

Criterios de exclusión: 1) artículos realizados fuera de los años 2010 a 2019; 2) que sean de idioma diferente a inglés y español; 3 ) acceso cerrado y/o con costo por visualización; 4) tema centrado en burnout; 5) con muestra de estudiantes universitarios; 6) países no pertenecientes a América latina.

Los trabajos obtenidos se registraron en hojas de cálculo y se analizaron en cuatro fases, las cuales sirvieron para elegir los artículos potencialmente relevantes para esta revisión, la descripción de dichas fases se realiza a continuación:

Fase 1: la estrategia de selección consistió en escribir la cadena de búsqueda en cada base de datos, con la opción de búsqueda avanzada y se aplicaron los siguientes filtros para la selección de documentos: 1) el periodo de tiempo de enero de 2010 a julio de 2019 y 2) que la búsqueda fuera en todos los campos. El registro de esta fase contiene los datos de identificación del documento hallado: título, autoría (s), año, país de publicación, DOI, tipo de recurso, de acceso y base de datos de donde se recuperó.

Fase 2: se mantuvieron los documentos de la fase anterior y se depuraron con una evaluación de tipo cualitativa (sí/no), quedando eliminados los trabajos duplicados. Estos criterios se añadieron al registro en la hoja de cálculo, quedando así menos documentos que en la primera fase.

Fase 3: los trabajos seleccionados se sometieron a un filtro de seis preguntas para su valoración en cuanto a su calidad metodológica, el aporte teórico y el aporte instrumental, y así poder ser candidatos a una lectura crítica completa. Cada pregunta tuvo un valor de dos puntos, si el artículo respondía totalmente a la pregunta, se le asignaba el número dos; si respondía parcialmente, un uno; y si no respondía o no tenía relación con la pregunta, cero. Para que el artículo avanzara a la siguiente fase cubrió un puntaje mínimo de nueve y máximo de doce.

Fase 4: en esta parte se encuentran los documentos potencialmente relevantes, organizados en una hoja de cálculo mediante un cuadro de análisis, el cual conserva el apartado de datos principales del artículo (Fase 1) y se añadieron columnas para conocer la revista a la que pertenece, además se revisó a fondo la parte metodológica, la muestra, el instrumento utilizado, los resultados, la discusión y las aportaciones.

\section{Aspectos éticos}

La presente revisión sistemática considera lo señalado por el Código Ético de la American Psychological Association (2017), por lo que se dio crédito a los trabajos de investigación consultados, teniendo en cuenta los derechos de autor de los artículos al realizar las citas y referencias respectivas.

\section{Codificación de datos}

De cada estudio se extrajeron las siguientes características: año de publicación, país de publicación, idioma, palabras clave, enfoque, diseño y alcance. En cuanto a la muestra se analizó el país de procedencia, tamaño, sexo, el rango de edad y la labor 
desempeñada; además, de los instrumentos, se recolectaron nombres, autorías, años de publicación y el objeto de evaluación.

\section{Análisis de datos}

Se utilizó el Programa Microsoft (c) Excel 2016 para la elaboración de la base de datos que incluye las fases de análisis, separadas por hojas, donde están contenidos todos los estudios seleccionados.

\section{Resultados}

Inicialmente se obtuvieron 258 trabajos, sin embargo, una vez aplicados los criterios de inclusión y exclusión y después de un proceso de depuración, únicamente seis de ellos cumplieron con las características requeridas para el análisis final (véase Figura 1)

\section{Características generales de las investigaciones}

Tras el análisis realizado se presentan los datos de identificación y las palabras clave de los artículos incluidos en la presente revisión. Los años de publicación de los estudios va de 2014 a 2018 y Colombia aparece como el principal impulsor dentro de estas publicaciones. Por otro lado, se observa una gran diversidad de palabras clave consideradas en cada artículo, aunque en orden de frecuencia de aparición son: Estrés laboral, Estrés, Salud y Factores de Riesgo Psicosocial (véase Tabla 1).

\section{Enfoque, diseño y alcance de los estudios}

En cuanto a la parte metodológica de los artículos incluidos en esta revisión, se observa que la principal característica de estos estudios es que son cuantitativos, con diseños no experimentales y transversales y en su mayoría con alcances descriptivos. Así mismo, se encontró la presen-

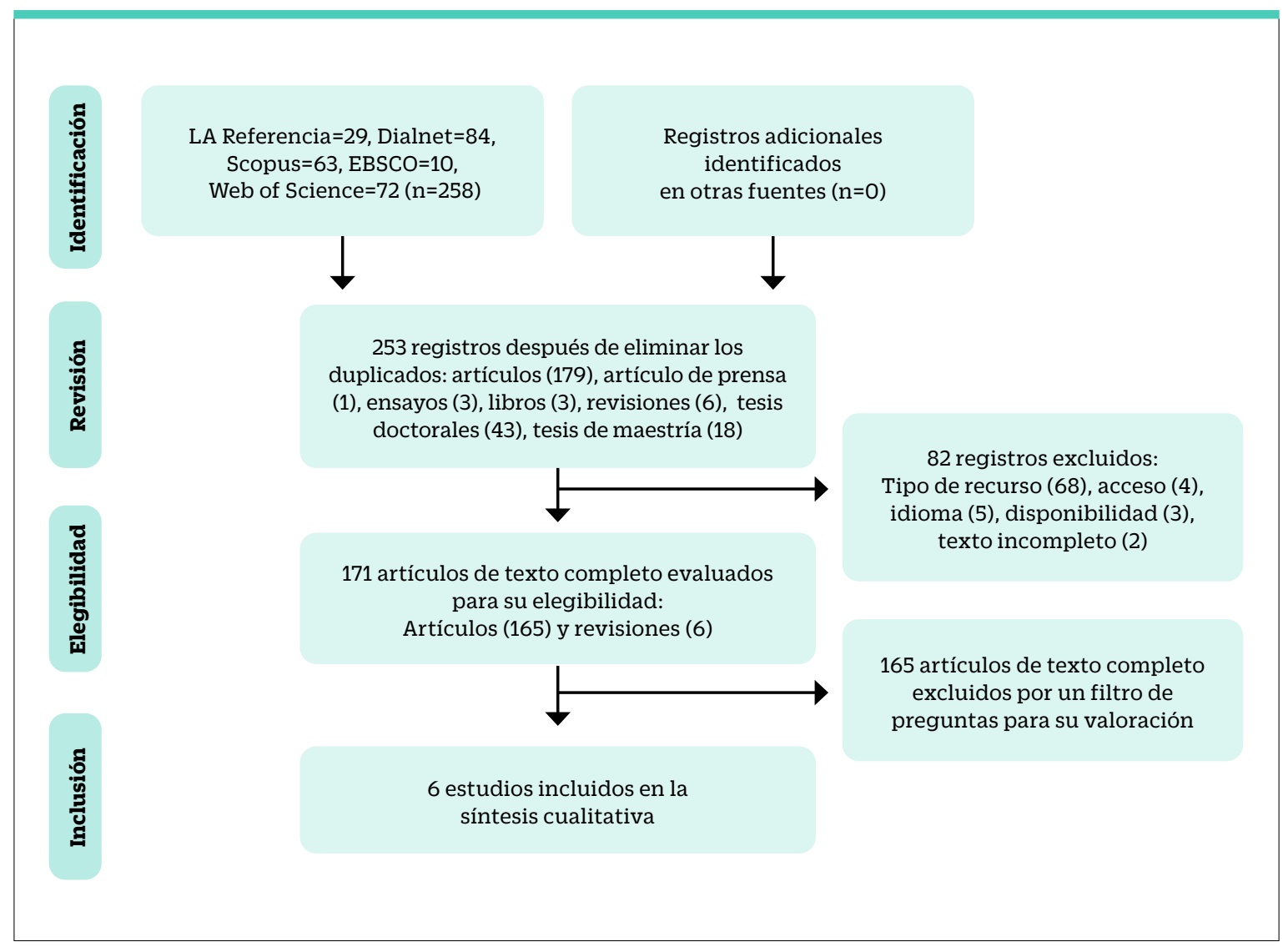

Figura 1. Algoritmo basado en el modelo PRISMA. 
Rojas-Solís, J., Flores-Meza, G. \& Cuaya-Itzcoatl, I.

Tabla 1

Principales características de las investigaciones revisadas

\begin{tabular}{|c|c|c|c|}
\hline Autoría & País de publicación & Idioma & Palabras clave \\
\hline $\begin{array}{l}\text { Martínez et al. } \\
(2018)\end{array}$ & Venezuela & Español & $\begin{array}{l}\text { Académicos, estímulos, salud, demandas, } \\
\text { salud ocupacional. }\end{array}$ \\
\hline $\begin{array}{l}\text { Matabanchoy, Lasso y } \\
\text { Pantoja } \\
(2017)\end{array}$ & Colombia & Español & $\begin{array}{l}\text { Estrés, instituciones educativas públicas, } \\
\text { salud, entorno laboral. }\end{array}$ \\
\hline $\begin{array}{l}\text { Romero, Beleño, Ucros, } \\
\text { Echeverría y Lasprilla } \\
\text { (2016) }\end{array}$ & Costa Rica & Español & $\begin{array}{l}\text { Estrés, factores-psicosociales, personal- } \\
\text { administrativo. }\end{array}$ \\
\hline $\begin{array}{l}\text { Unda et al. } \\
(2016)\end{array}$ & España & Español & $\begin{array}{l}\text { Factores de riesgo psicosocial, trabajo, } \\
\text { docentes universitarios, validación } \\
\text { psicométrica, medición. }\end{array}$ \\
\hline $\begin{array}{l}\text { Gómez, Perilla y Hermosa } \\
\text { (2015) }\end{array}$ & Colombia & Español & $\begin{array}{l}\text { Trabajo y familia, estrés laboral, malestar } \\
\text { psicológico, burnout, psicología de la salud. }\end{array}$ \\
\hline $\begin{array}{l}\text { Millán, García-Álvarez y } \\
\text { D’Aubeterre } \\
\text { (2014) }\end{array}$ & Colombia & Español & $\begin{array}{l}\text { Inteligencia emocional, flujo de trabajo, estrés } \\
\text { en el trabajo, bienestar. }\end{array}$ \\
\hline
\end{tabular}

cia de un estudio con diseño no experimental y transversal, con alcance descriptivo y además con aportaciones instrumentales, pues se trata de la validación de una escala (Unda et al., 2016), lo anterior se puede visualizar en la Tabla 2 . .

\section{Participantes}

Referente a las muestras, se puede observar que proceden principalmente de Colombia. Asimismo, se presenta su descripción donde existen variaciones respecto al tamaño de las muestras donde, la más pequeña es de 196 personas, comparada con la de 957 docentes, que es la más grande. En cuanto a la distribución de los participantes, por sexo, los hombres fueron quienes participaron más; mientras que la edad mínima presentada en los estudios es de 21 años y la máxima registrada es de 76, y respecto a la variable labor, hay más intervención en docentes seguido del personal administrativo (ver Tabla 3).

\section{Características de los instrumentos}

Respecto a los instrumentos utilizados en cada artículo analizado, se detectó un total de 15, de los cuales 6 son cuestionarios, 5 escalas, un inventario, una encuesta, una prueba y una batería. En cuanto a la antigüedad de los instrumentos hay que destacar que la mayoría de ellos presenta un

Tabla 2

Enfoque, diseño y alcance de los estudios revisados

\begin{tabular}{llll} 
Autoría & Enfoque & Diseño & Alcance \\
\hline Martínez et al., 2018 & Cuantitativo & No Exp/Transversal & $\begin{array}{c}\text { Correlacional/ } \\
\text { Descriptivo }\end{array}$ \\
\hline Matabanchoy et al., 2017 & Cuantitativo & No Exp/Transversal & Descriptivo \\
\hline Romero et al., 2016 & Cuantitativo & No Exp/Transversal & Descriptivo \\
\hline Unda et al., 2016 & Cuantitativo & No Exp/Transversal & Descriptivo \\
\hline Gómez et al., 2014 & Cuantitativo & No Exp/Transversal & Correlacional \\
\hline Millán et al., 2014 & Cuantitativo & No Exp/Transversal & Explicativo \\
\hline
\end{tabular}


Tabla 3

Principales características de las muestras de las investigaciones revisadas

\begin{tabular}{|c|c|c|c|c|c|}
\hline \multirow{2}{*}{ Autoría } & \multicolumn{5}{|c|}{ Muestra } \\
\hline & Procedencia & Tamaño & Sexo & Rango de edad & Labor \\
\hline $\begin{array}{l}\text { Martínez et al., } \\
2018\end{array}$ & México & 957 docentes & $\begin{array}{l}61 \% \text { hombres y } \\
39 \% \text { mujeres } \\
\text { (587 hombres y } \\
370 \text { mujeres) }\end{array}$ & 26 a 76 años & Docentes \\
\hline $\begin{array}{l}\text { Matabanchoy et } \\
\text { al., } 2017\end{array}$ & Colombia & 196 personas & $\begin{array}{l}65 \% \text { mujeres y } \\
35 \% \text { hombres }\end{array}$ & 21 a 65 años & $\begin{array}{l}\text { Directivos, } \\
\text { administrativos, } \\
\text { docentes y personal de } \\
\text { apoyo }\end{array}$ \\
\hline $\begin{array}{l}\text { Romero et } \\
\text { al., } 2016\end{array}$ & Colombia & 227 trabajadores & $\begin{array}{l}157 \text { mujeres y } 70 \\
\text { hombres }\end{array}$ & $\mathrm{N} / \mathrm{E}$ & Personal administrativo \\
\hline Unda et al., 2016 & México & 500 docentes & $\begin{array}{l}331 \text { hombres y } \\
168 \text { mujeres }\end{array}$ & 21 a 76 años & Profesorado \\
\hline $\begin{array}{l}\text { Gómez et } \\
\text { al., } 2014\end{array}$ & Colombia & 302 académicos & $\begin{array}{l}57.3 \% \text { hombres } \\
\text { y } 42.7 \% \text { mujeres }\end{array}$ & $\begin{array}{l}\text { Edad promedio } \\
\text { de } 42.3 \text { años }\end{array}$ & Académicos \\
\hline $\begin{array}{l}\text { Millán et } \\
\text { al., } 2014\end{array}$ & Venezuela & 199 docentes & $\begin{array}{l}67.34 \% \text { mujeres } \\
\text { y } 32.66 \% \\
\text { hombres }\end{array}$ & $\begin{array}{l}\text { Entre } 22 \text { y } 73 \\
\text { años }\end{array}$ & $\begin{array}{l}\text { Profesores de pre grado } \\
\text { y pos grado }\end{array}$ \\
\hline
\end{tabular}

Nota. $N / E=$ No especifica.

periodo de publicación mayor a los últimos 10 años. Por otro lado, se encontró que aquellos que evalúan el estrés y los factores de riesgo laborales son los más utilizados y son validados para población de países latinoamericanos. Los datos y descripción de cada uno se muestran en la Tabla 4.

\section{Discusión}

Esta revisión sistemática tuvo por objetivo analizar los principales aspectos metodológicos de estudios, publicados entre los años 2010 y 2019, sobre estrés laboral en docentes, administrativos y personal de apoyo que trabaja en universidades públicas. Así mismo, identificar los instrumentos utilizados para medir y/o evaluar el estrés laboral en personal universitario.

Los países impulsores de las publicaciones analizadas fueron: España, Costa Rica, Perú, Venezuela y principalmente Colombia, país que parece mostrar una preocupación por la producción científica y por estudios sobre diferentes problemas relacionados con el entorno nacional y regional, desde finales del siglo pasado (Pérez, 2013).

Un hallazgo importante fue la diversidad de palabras clave consideradas en cada artículo, donde se mencionan a académicos, administrativos y docentes, pero omitiendo personal de apoyo, el cual también es parte de la universidad. Sin embargo, estaría incluido en el grupo de los llamados "nodocentes", quienes por tiempo han sido invisibilizados e incluso excluidos de decisiones fundamentales (Astorga, 2018).

La totalidad de los artículos analizados fueron elaborados desde un enfoque cuantitativo con diseños no experimentales y transversales, en su mayoría con alcance descriptivo, lo cual implica aportaciones válidas desde un punto de vista exploratorio, descriptivo, diagnóstico y localizado, pero al mismo tiempo las limitaciones propias de este tipo de diseños que impiden, por ejemplo, la inferencia causal entre variables o el seguimiento del fenómeno a través del tiempo.

En cuanto a las muestras de los estudios, se observaron variaciones en el tamaño y la distribución de los participantes, así se encontró que ambos sexos participaron, sin embargo, se notó mayor presencia de hombres. Esto podría deber- 
Rojas-Solís, J., Flores-Meza, G. \& Cuaya-Itzcoatl, I.

Tabla 4

Características de los instrumentos implementados en las investigaciones revisadas

\begin{tabular}{|c|c|c|c|}
\hline \multirow{2}{*}{ Autoría } & \multicolumn{3}{|c|}{ Instrumento } \\
\hline & Nombre & Autoría & Qué evalúa \\
\hline $\begin{array}{l}\text { Martínez et al., } \\
2018\end{array}$ & $\begin{array}{l}\text { 1.Encuesta adaptada del } \\
\text { Programa de Evaluación y } \\
\text { Seguimiento de la Salud de } \\
\text { los Trabajadores (PROESSAT) } \\
\text { 2.Inventario DASS } 21 \\
\text { 3.Prueba Yoshitake }\end{array}$ & $\begin{array}{l}\text { 1.Noriega, Franco, Martínez, } \\
\text { Villegas, Alvear y López, } 2001 \\
\text { 2.Daza, Novy, Stanley y } \\
\text { Averill, } 2002 \\
\text { 3.Barrientos, Martínez y } \\
\text { Méndez, } 2004\end{array}$ & $\begin{array}{l}\text { 1.N/E } \\
\text { 2.Valora ansiedad, depresión y } \\
\text { estrés (validado con población } \\
\text { latina) } \\
\text { 3.Mide fatiga (validado en } \\
\text { población mexicana) }\end{array}$ \\
\hline $\begin{array}{l}\text { Matabanchoy et } \\
\text { al., } 2017\end{array}$ & $\begin{array}{l}\text { 1.Cuestionario para la } \\
\text { evaluación del estrés, tercera } \\
\text { versión, de la Batería de } \\
\text { Riesgos Psicosociales }\end{array}$ & 1.Villalobos, 2010 & $\begin{array}{l}\text { 1.Síntomas del estrés } \\
\text { (fisiológicos, de } \\
\text { comportamiento } \\
\text { social, laboral, intelectuales y } \\
\text { psicoemocionales) }\end{array}$ \\
\hline $\begin{array}{l}\text { Romero et } \\
\text { al., } 2016\end{array}$ & $\begin{array}{l}\text { 1.Cuestionario para la } \\
\text { evaluación del estrés } \\
\text { 2.Batería de Instrumentos } \\
\text { para la Evaluación de FRP }\end{array}$ & $\begin{array}{l}\text { 1.Castillo y Villalobos, } 1996 \\
\text { 2.Ministerio de la Protección } \\
\text { Social (MPS), } 2010\end{array}$ & $\begin{array}{l}\text { 1.Estrés en tres categorías: alto, } \\
\text { medio y bajo } \\
\text { 2.FRP en las instituciones }\end{array}$ \\
\hline Unda et al., 2016 & $\begin{array}{l}\text { 1.Escala para valorar los FRP } \\
\text { en el trabajo de profesores } \\
\text { universitarios }\end{array}$ & $\begin{array}{l}\text { 1.Unda, Uribe, Jurado, García, } \\
\text { Tovalín y Juárez, } 2016\end{array}$ & $\begin{array}{l}\text { 1.FRP a los que está expuesto el } \\
\text { profesorado universitario }\end{array}$ \\
\hline $\begin{array}{l}\text { Gómez et } \\
\text { al., } 2015\end{array}$ & $\begin{array}{l}\text { 1.Cuestionario del Contenido } \\
\text { del Trabajo (JCQ) } \\
\text { 2.El Cuestionario General } \\
\text { de Salud (General Health } \\
\text { Questionnaire, GHQ-28) } \\
\text { 3.Escala de transferencias } \\
\text { familia-trabajo de Grzywacz } \\
\text { y Marks } \\
\text { 4.Cuestionario Maslach } \\
\text { Burnout Inventory (MBI-G) }\end{array}$ & $\begin{array}{l}\text { 1.Gómez, } 2011 \\
\text { 2.N/E } \\
\text { 3.Pérez, } 2004 \\
\text { 4.Gil-Monte, } 2002\end{array}$ & $\begin{array}{l}\text { 1.FRP laborales } \\
\text { 2.Síntomas Psicosomáticos, } \\
\text { Ansiedad y Problemas de Sueño, } \\
\text { Depresión y Disfunción Social } \\
\text { 3.El conflicto y la facilitación } \\
\text { familia-trabajo a través de } \\
\text { cuatro dimensiones } \\
\text { 4.Agotamiento Emocional, } \\
\text { Cinismo y Eficacia Profesional. }\end{array}$ \\
\hline $\begin{array}{l}\text { Millán et } \\
\text { al., } 2014\end{array}$ & $\begin{array}{l}\text { 1.Escala de Bienestar } \\
\text { Psicológico (EBP) } \\
\text { 2.Escala de Disposición a Fluir } \\
\text { en el Trabajo (EDFT-3), tercera } \\
\text { versión } \\
\text { 3.Trait Meta-Mood Scale } 24 \\
\text { (TMMS-24) } \\
\text { 4.Inventario de Percepción } \\
\text { de Estresores en Docentes } \\
\text { Universitarios (IPED-U) }\end{array}$ & $\begin{array}{l}\text { 1.Millán y D'Aubeterre, } 2011 \\
\text { 2.Millán y D'Aubeterre, } 2012 \text {, } \\
2013 \\
\text { 3.Fernández-Berrocal, } \\
\text { Extremera y Ramos, } 2004 \\
\text { 4.Álvarez, D'Aubeterre y } \\
\text { Ramírez, } 2011\end{array}$ & $\begin{array}{l}\text { 1.Grado de felicidad y } \\
\text { satisfacción con la vida que } \\
\text { percibe la persona } \\
\text { 2.Grado en que un trabajador } \\
\text { se encuentra en un estado } \\
\text { psicológico óptimo (términos de } \\
\text { motivación, disfrute personal } \\
\text { de la tarea y el desempeño de } \\
\text { su rol) } \\
\text { 3.Atención, claridad y } \\
\text { reparación. } \\
\text { 4.El grado de incidencia } \\
\text { de estrés en docentes } \\
\text { universitarios venezolanos }\end{array}$ \\
\hline
\end{tabular}

Nota. $N / E=$ No especifica, $F R P=$ Factores de riesgo psicosocial.

se a que, en América Latina y el Caribe, ha crecido la tasa de participación laboral de las mujeres. Sin embargo, aún hay una diferencia de $25 \%$ respecto a los hombres, originando que las tasas de mujeres desempleadas sean más elevadas que las de los hombres (Poltorak, 2018). Respecto a la edad de los participantes, donde la mínima registrada en los estudios fue de 21 años y la máxima de 76, es pertinente señalar que ese rango de edad abarca diferentes etapas del ciclo vital por lo que se invita a la cautela en su interpretación, sobre todo si se considera que los eventos estresantes a lo largo 
de la vida tienen significados e impactos diferentes (Pearlin, 2010) sin olvidar que en cada etapa se tiene una construcción distinta de las representaciones sociales sobre los elementos que debe contener la calidad de vida (Razo et al., 2018) y el trabajo, pues éste suele determinar la autoestima, la autorregulación, la autoeficacia y las oportunidades sociales (Siegrist, 1996).

Se evidenció que los estudios se enfocan más en el personal docente, seguido de los administrativos, por lo que existe poca (o casi nula) información sobre investigaciones dirigidas a personal que trabaja como apoyo en las universidades, sector que parece haber sido relegado. Se trata de una tendencia que ya ha sido apuntada por otros autores pues parece que las investigaciones vinculadas con la universidad se siguen focalizando, generalmente, en docentes (Luy-Montejo et al., 2019) y, en algunos casos, en alumnos (Marsollier et al., 2014).

Ahora bien, en cuanto a los instrumentos utilizados en las publicaciones, solo cuatro cumplen con el objetivo de la presente revisión sistemática, es decir, la evaluación concreta del estrés laboral: 1) el inventario DASS 21, que valora ansiedad, depresión y estrés; 2) la tercera versión de la Batería de Riesgos Psicosociales, la cual identifica síntomas fisiológicos, de comportamiento social y laboral, intelectuales y psicoemocionales del estrés; 3) el Cuestionario de Estrés, donde el fenómeno se estima en tres categorías: alto, medio y bajo; y 4) el Inventario de Percepción de Estresores en Docentes Universitarios (IPED-U), el cual evalúa el grado de incidencia de diferentes fuentes de estrés. Además, se pudo observar gran variedad de instrumentos para la evaluación del fenómeno, en ese sentido los más sobresalientes fueron el Cuestionario para la evaluación del estrés y el Cuestionario de Maslach Burnout Inventory (MBI). En este segundo caso, es importante mencionar que el MBI no está destinado para evaluar el estrés laboral, sino el burnout, el cual es una respuesta prolongada y evolucionada del estrés laboral (Olivares, 2017; Rodríguez \& Rivas, 2011), por lo que las herramientas utilizadas para analizar dicho síndrome son difíciles de extrapolar a niveles de estrés general o del día a día(García-Unanue et al., 2017).
$\mathrm{Al}$ respecto es preciso señalar que en la actualidad el estrés, considerado como un término complejo, aún no cuenta con un consenso en su delimitación (Silva-Ramos et al., 2020). Esta dificultad para definir el estrés no radica tanto en la diversidad de significados como en la existencia de los usos que se le han dado, el popular y el científico, pues aunque todo el mundo cree saber de qué se trata, poca gente parece capaz de delimitar con exactitud su significado (Rivera, 2010). En ese mismo sentido, es preciso señalar que el estrés laboral y el burnout mantienen inseparables lazos de unión (Barraca, 2010) que derivan en problemas teóricos que aún se tienen sobre el constructo, generando muchas veces un uso indistinto de los conceptos en la investigación empírica (Cajachagua et al., 2015). Por ello, se considera importante tener en cuenta las implicaciones teóricas y metodológicas, el diagnóstico del fenómeno, la interpretación de los resultados obtenidos y el establecimiento de líneas de investigación más precisas.

Respecto a las limitaciones de este estudio se encuentran la rigurosidad de criterios de inclusión, así como la especificación de la muestra perteneciente a América Latina, lo que pudo haber influido en el reducido número de estudios obtenidos para esta revisión, además del interés únicamente en docentes, administrativos y personal de apoyo, sectores importantes de la universidad pública, sin olvidar el interés específico en el estrés laboral. Añadido a ello, los estudios analizados solo fueron aquellos de libre acceso.

Finalmente, en cuanto a las futuras líneas de investigación, se considera pertinente delimitar el concepto de estrés laboral del de burnout, para no seguir contribuyendo a confusiones conceptuales y terminológicas. Asimismo se sugiere dirigir estudios hacia diferentes sectores de la universidad, por ejemplo, el personal de apoyo y el personal administrativo, en los cuales haya una detección temprana sobre el fenómeno en cuestión y, si es preciso, buscar la disminución del estrés o mejor aún prevenir dicha enfermedad en los trabajadores. Si bien es cierto que muchas veces no se puede eliminar el estrés, sí se pueden desarrollar habilidades y estrategias para manejarlo y controlarlo (Durán, 2010). Además, es preciso considerar de 
Rojas-Solís, J., Flores-Meza, G. \& Cuaya-Itzcoatl, I.

forma teórica y metodológica las condiciones actuales del personal universitario, en especial el confinamiento debido a la pandemia, es así como el teletrabajo (Rodríguez, 2017; Vicente et al., 2018) que emerge cada vez con más frecuencia e importancia (Muñoz et al., 2018). Se trata de una modalidad de trabajo que ha presentado beneficios (Valle, 2018), pero también consecuencias indeseables, como el tecnoestrés (CCuervo et al., 2018), un fenómeno que podría ser materia de investigación en trabajos venideros. Por último, se enfatiza la pertinencia de implementar programas que promuevan la salud y el bienestar físico y psicológico de las personas que laboran en la Universidad.

\section{Conflictos de interés}

No existen conflictos de interés.

\section{Referencias}

*Artículos incluidos en la revisión sistemática

Acosta, A. (2019). El poder universitario en América Latina. Revista Mexicana de Sociología, 81(1), 117-144. Recuperado de http://www.scielo.org.mx/pdf/rms/ v81n1/0188-2503-rms-81-01-117.pdf

Álvarez, J.C., D’Aubeterre, M. E., \& Ramírez, T. (2011). Construcción de un inventario de percepción de estresores en docentes universitarios. En Blanco, C. (Coord.). Investigación Educativa: Venezuela en Latinoamérica Siglo XXI (Parte II). (pp. 93 - 116). Caracas, Venezuela: Centro de Investigaciones Educativas (CIES) de la Escuela de Educación de la Universidad Central de Venezuela (UCV). Recuperado de http://www.ucv.ve/organizacion/ f a cultades/facultad-de-humanidades - y educacion/centros-de-investigacion/centro-deinvestigaciones-educativas-cies/publicaciones/ libros/investigacion-en-educacion-venezuela-enlatinoamerica-parte-ii.html

Alves, D., Cirera, Y., \& Carlos, A. (2013). Vida con calidad y calidad de vida en el trabajo. Invenio, 16(30), 145-163. Recuperado de https://www.redalyc.org/ pdf/877/87726343010.pdf

Alvites-Huamaní, C. G. (2019). Estrés docente y factores psicosociales en docentes de Latinoamérica, Nor- teamérica y Europa. Propósitos y Representaciones, 7(3), 141-178. http://doi.org/10.20511/pyr2019. v7n3.393

American Psychological Association. (2017). Ethical Principles of Psychologists and Code of Conduct. Washington, DC: Autor. Recuperado de https:// www.apa.org/ethics/code/ethics-code-2017.pdf

Aparicio, C., \& Sepúlveda, F. (2019). Trabajo colaborativo docente: nuevas perspectivas para el desarrollo docente. Psicología Escolar e Educacional, (23), 1-7. http://doi.org/10.1590/2175-35392019017926

Astorga, S. (2018). Desafíos de la capacitación del personal nodocente en el sistema universitario argentino. Integración y conocimiento, 2(7), 150-166. Recuperado de https://revistas.unc.edu.ar/index. php/integracionyconocimiento/article/view/21931

Avargues, M. L., \& Borda, M. (2010). Estrés laboral y síndrome de burnout en la universidad: análisis descriptivo de la situación actual y revisión de las principales líneas de investigación. Anuario de psicología clínica y de la salud, 6, 73-78. Recuperado de http://institucionales.us.es/apcs/doc/APCS_6_ esp_73-78.pdf

Bairero, M. E. (2017). El estrés y su influencia en la calidad de vida. Multimed Revista Médica, 21(6), 971-982. Recuperado de http://www.revmultimed.sld.cu/ index.php/mtm/article/view/688

Barraca, J. (2010). Emociones negativas en el profesorado universitario: burnout, estrés laboral y mobbing. EduPsykhé, 9(1), 85-99. Recuperado de http://repositorio.ucjc.edu/ bitstream/handle/20.500.12020/22/C00042387. pdf?sequence $=1$

Barrientos, P. (2016). La naturaleza de la formación docente. Horizonte de la ciencia, 6(11), 169-177. Recuperado de http://revistas.uncp.edu.pe/index.php/ horizontedelaciencia/article/view/331

Barrientos, T., Martínez, S., \& Méndez, I. (2004). Validez de constructo, confiabilidad y punto de corte de la Prueba de Síntomas Subjetivos de Fatiga (Yoshitake) en trabajadores mexicanos, Salud Pública de México, 46(6): 516-523. Recuperado de http://www.scielo.org.mx/scielo.php?script=sci_ arttext\&pid=S0036-36342004000600006

Beltrán, O. (2005). Revisiones sistemáticas de la literatura. Revista Colombiana de Gastroenterología, 20(1), 60-69. Recuperado de http://www.scielo.org.co/pdf/ rcg/v20n1/v20n1a09.pdf

Berrio, N., \& Mazo, R. (2011). Estrés académico. Revista de 
Psicología Universidad de Antioquia, 3(2), 65-82. Recuperado de https://revistas.udea.edu.co/index. $\mathrm{php} / \mathrm{psicologia/article/view/11369/10646}$

Birolim, M. M., Mesas, A. E., González, A., Geremias, H., Fernandez, M., \& Andrade, S. M. (2019). Job strain among teachers: associations with occupational factors according to social support. Ciência \& Saúde Coletiva, 24(4), 1255-1264. https://doi. org/10.1590/1413-81232018244.08542017

Botero, C. C. (2012). Riesgo psicosocial intralaboral y "burnout" en docentes universitarios de algunos países latinoamericanos. Cuadernos de Administración, 28(48), 117-132. Recuperado de http://www.redalyc. org/articulo.oa?id=225025860002

Cajachagua, M., Salinas, S. A., \& Carranza, R. (2014). Efectividad del programa de ejercicios físicos "Muévase por su salud" sobre las medidas antropométricas y el estrés laboral del personal de la UPeU filial Tarapoto, 2014. Revista de Investigación Universitaria, 3(2), 46-51. Recuperado de https:// www.researchgate.net/publication/326302794

Carpio, I., Bravo, G., Campos, N., Padilla, A., Banegas, T., \& Méndez, L. (2017). Estrés laboral en docentes, administrativos $\mathrm{y}$ trabajadores universitarios. Revista Electrónica de Psicología Iztacala, 20(3), 145-164. Recuperado de https://www.medigraphic. com/pdfs/epsicologia/epi-2017/epi173i.pdf

Castillo, M., \& Villalobos, F. (1996). Cuestionario para la evaluación del estrés. Bogotá: Ministerio de Trabajo y Seguridad Social.

Collado, P. A., Soria, C., Canafoglia, E., \& Collado, S. A. (2016). Condiciones de trabajo y salud en docentes universitarios y de enseñanza media de Mendoza, Argentina: entre el compromiso y el desgaste emocional. Salud colectiva, 12(2), 203220. Recuperado de https://www.redalyc.org/ pdf/731/73146051005.pdf

Cuervo, T., Orviz, N., Arce, S., \& Fernández, I. (2018). Tecnoestrés en la Sociedad de la Tecnología y la Comunicación: revisión bibliográfica a partir de la Web of Science. Archivos de Prevención de Riesgos Laborales, 21(1), 18-25. https://doi.org/10.12961/ aprl.2018.21.01.4

Daza, P., Novy, D., Stanley, M., \& Averill, P. (2002). The Depression Anxiety Stress Scale-21: Spanish Translation and Validation with a Hispanic. Journal of Psychopathology and Behavioral Assessment, 24, 195-205. https://doi. org/10.1023/A:1016014818163
Durán, C., Atlante, M., \& Giordano, D. (diciembre, 2010). El clima organizacional en la universidad: modelo de medición para el personal de apoyo universitario. $X$ Coloquio Internacional sobre Gestión Universitaria en América del Sur, Mar del Plata, Argentina. Recuperado de https://repositorio.ufsc.br/ bitstream/handle/123456789/96677/DURAN. pdf?sequence $=1$

Durán, M. M. (2010). Bienestar psicológico: el estrés y la calidad de vida en el contexto laboral. Revista Nacional de Administración, 1(1), 71-84. Recuperado de https://revistas.uned.ac.cr/index.php/rna/ article/view/285/146

Fernández-Berrocal, P., Extremera, N., \& Ramos, N. (2004). Validity and reliability of the spanish modified version of the trait meta-mood scale. Psychological Reports, 94(1), 751-755. Recuperado de http:// emotional.intelligence.uma.es/pdfs/spanish\%20 tmms.pdf

Figueroa, G. J., Encinas, D. I., Félix, P. M., \& Vega, A. L. (2019). Comparativo de la funcionalidad institucional de Educación Superior en México y Colombia. Revista de Estudios y Experiencias en Educación, 18(38), 7186. http://doi.org/10.21703/rexe.20191838figueroa5

Ganster, D. C., \& Rosen, C. C. (2013). Work Stress and Employee Health: A Multidisciplinary Review. Journal of Management, 39(5), 1085-1122. https:// doi.org/10.1177/0149206313475815

García, B. R., Maldonado, S. E., Ramírez, M. C., \& Lozano, M. (2013). Diagnóstico del nivel de estrés laboral y su relación con el apoyo social percibido en trabajadores de la salud mexicanos. Global Conference on Business and Finance Proceedings, 8(1), 1350-1358. Recuperado de https://www. researchgate.net/publication/293488574

García, J. L. (2016). Estudio del estrés laboral en el personal administrativo y trabajadores de la universidad de cuenca. Cuenca 2015. (Tesis de Maestría). Universidad de Cuenca, Ecuador. Recuperado de https://dspace.ucuenca.edu.ec/ bitstream/123456789/24644/1/Tesis.pdf

García, M. A., González, R., Aldrete, M. G., Acosta, M., \& León, S. G. (2014). Relación entre Calidad de Vida en el Trabajo y Síntomas de Estrés en el Personal Administrativo Universitario. Ciencia \& trabajo, 16(50), 97-102. http://doi.org/10.4067/S071824492014000200007

García-Unanue, J., León, M., Colino, E., \& Gallardo, E. (2017). Gestión del estrés en entornos laborales 
Rojas-Solís, J., Flores-Meza, G. \& Cuaya-Itzcoatl, I.

y actividad física. Revisión sobre instrumentos de medición. Kronos, 16(1), 1-8. Recuperado de https://abacus.universidadeuropea.es/ bitstream/handle/11268/6552/Kronos_2017_1_2. pdf? sequence $=2 \&$ is Allowed $=y$

Gil-Monte, P. (2002). Validez factorial de la adaptación al español del Maslach Burnout InventoryGeneral Survey. Salud Pública de México, 44(1), 33-40. Recuperado de http://www. scielo.org.mx/scielo.php?script =s ci arttext\&pid=S0036-36342002000100005

Gómez, V. (2011). Assessment of psychosocial stressor at work: Psychometric properties of the Spanish version of the JCQ (Job Content Questionnaire) in Colombian workers. Revista Latinoamericana de Psicología, 43(2), 125-138. Recuperado de https://dialnet.unirioja.es/servlet/ articulo?codigo $=3756343$

Gómez, V., Perilla, L. E., \& Hermosa, A. M. (2015). Moderación de la relación entre la tensión laboral y la insatisfacción de los profesores universitarios: el papel del conflicto y la facilitación entre el trabajo y la familia. Revista Colombiana de Psicología, 24(1), 185-201. https://doi.org/10.15446/rcp.v24n1.42081

Gonzáles, E. (2014). Clima psicológico universitario desde la perspectiva del personal administrativo de la Universidad Nacional Toribio Rodríguez de Mendoza de Amazonas, Chachapoyas. Revista Tzhoecoen, 6(1), 199-212. Recuperado de http:// revistas.uss.edu.pe/index.php/tzh/article/ view/27/26

González, M. L., \& Codagnone, T. (diciembre, 2010). La importancia de la tecnoestructura y el staff de apoyo en la organización de las instituciones de educación superior. $X$ Coloquio Internacional sobre Gestión Universitaria en América del Sur, Mar del Plata, Argentina. Recuperado de https://repositorio. ufsc.br/xmlui/bitstream/handle/123456789/96752/ GONZALEZ.pdf?sequence $=1$

González, L., \& Orta, Y. (2016). La cultura organizacional y el síndrome de desgaste profesional en profesores universitarios. Revista Chakiñan, (1), 14-30. https:// doi.org/10.37135/chk.002.01.02

Hermosa, A. M., \& Perilla, L. E. (2015). Retos investigativos en psicología de la salud ocupacional: el estrés laboral. Facultad Nacional de Salud Pública, 33(2), 252-261. http://doi.org/10.17533/udea.rfnsp.v33n2a12

Hessels, J., Rietveld, C.A., \& Zwan, P. (2017). Self-employment and work-related stress: The mediating role of job control and job demand. Journal of Business Venturing, 32(2), 178-196. http://doi.org/10.1016/j. jbusvent.2016.10.007

Huanco, D. (2019). La universidad como centro formador de investigadores. Revista Médica Hospital Hipólito, 12(2), 66-67. Recuperado de http://revista. hospitaltacna.gob.pe/index.php/revista2018/ article/download/124/101

Karam, J. M., Parra, C., Urrego, G., \& Castillo, C. (2019). Estrés en el colegio. Dos variables para reflexionar. Revista tesis Psicológica, 14(1), 1-27. Recuperado de https:// dialnet.unirioja.es/descarga/articulo/7150889.pdf

Karasek, R. (1979). Job demands, job decision latitude and mental strain: Implications for job redesign. Administrative Science Quaraterly, 24(2), 285-306.

Londoño, L. F. (2019). Análisis de las características del estrés laboral, según datos sociodemográficos, en el personal de la Corporación Universitaria Lasallista de Caldas Antioquia en el año 2016. Fides et Ratio - Revista de Difusión cultural y científica de la Universidad La Salle en Bolivia, 17(17), 39-60. Recuperado de http://www.scielo.org.bo/pdf/rfer/ v17n17/v17n17_a04.pdf

Luy-Montejo, C., Teves, J., Rojas, W., Ramos, T., Lazo de la Vega, A., Arias, D., Aguinaga-Villegas, D., \& GálvezSuarez, E. (2019). Análisis de la producción científica latinoamericana sobre estrés docente (2010 - 2018). Propósitos y Representaciones, 7(3), 01-11. http:// doi.org/10.20511/pyr2019.v7n3.392

Marsollier, R. G., \& Aparicio, M. (2014). Universidad y factores de riesgo laboral. Una aproximación al desgaste profesional en empleados universitarios. Revista de Psicología-Universidad Viña del Mar, 3(6), 24-33. Recuperado de http://sitios.uvm.cl/ revistapsicologia/revista/06.06.universidad.pdf

Martínez, S., Castro, G., Sánchez, C. D., Martínez, C. R., Irigoyen, F. D., Inchaustegui, L., \& Zamora, M. (2018). Programa de estímulos económicos y daños a la salud en académicos de seis universidades mexicanas. Salud de los trabajadores, 26(2), 98-111. Recuperado de https://dialnet.unirioja.es/descarga/ articulo/6955479.pdf

Matabanchoy, S. M., Lasso, E. N., \& Pantoja, M. A. (2017). Estrés en funcionarios de Instituciones Educativas Públicas. Revista Psicoespacios, 11(19), 5775. Recuperado de http://revistas.iue.edu.co/ revistasiue/index.php/Psicoespacios/article/ download/939/1212

McCarthy, C. J. (2019). Teacher stress: Balancing demands 
and resources. Phi Delta Kappan, 101(3), 8-14. https://doi.org/10.1177/0031721719885909

Meurs, J. A., \& Perrewé, P. L. (2011). Cognitive Activation Theory of Stress: An Integrative Theoretical Approach to Work Stress. Journal of Management, 37(4), 1043-1068. https://doi. org/10.1177/0149206310387303

Mejía, M. R. (2015). Reconfiguración del capitalismo globalizado y resistencias desde América Latina. Nómadas, 43, 149-165. Recuperado de https:// dialnet.unirioja.es/descarga/articulo/5428005.pdf

Ministerio de la Protección Social, MPS. (2010). Batería de Instrumentos para la Evaluación de Factores de Riesgo Psicosocial, Resolución 2646 de 2008. Recuperado de http://fondoriesgoslaborales.gov. co/documents/Publicaciones/Estudios/Bateriariesgo-psicosocial-1.pdf

Millán, A., \& D'Aubeterre, M. E. (2011). Validación de la escala de bienestar psicológico en una muestra multiocupacional venezolana. Revista CES Psicología, 4(1), 52-71. Recuperado de https:// www.researchgate.net/publication/215688975_ Validacion_de_la_Escala_de_Bienestar_Psicologico_ en_una_muestra_multiocupacional_venezolana

Millán, A., \& D’Aubeterre, M. E. (2012). Confirmación de la estructura factorial de la Escala de Disposición a Fluir en el Trabajo. En A. Salcedo (Ed.), Estadística en la investigación: competencia transversal en la formación universitaria (pp. 117-144). Caracas: Programa de Cooperación Interfacultades, Vicerrectorado Académico, Universidad Central de Venezuela. Recuperado de http://saber.ucv.ve/ jspui/handle/123456789/4646

Millán, A., García-Álvarez, D., \& D’Aubeterre, M. E. (2014). Efecto de la inteligencia emocional y flujo en el trabajo sobre estresores y bienestar psicológico: Análisis de ruta en docentes. Revista Colombiana de Psicología, 23(1), 207-228. Recuperado de https:// www.researchgate.net/publication/273476985

Moher, D., Liberati, A., Tetzlaff, J., Altman, D. G., \& The PRISMA Group. (2009). Preferred Reporting Items for Systematic Reviews and Meta-Analyses: The PRISMA Statement. PLOS Medicine 6(6). https://doi. org/10.1371/journal.pmed.1000097

Muñoz, A., Amórtegui, V., \& Esguerra, G. (2018) El teletrabajo. Justicia Juris, 14(1), 25-31. http://doi.org/10.15665/ rj.v14i1.1976

Nava, C., Orihuela, L., \& Vega, C. (2016). Relación entre estrés laboral y estrés cotidiano. Revista Electrónica de
Psicología Iztacala, 19(2), 558-568. Recuperado de https://www.medigraphic.com/cgi-bin/new/ resumen.cgi?IDARTICULO=73902

Noriega, M., Franco, J., Martínez, S., Villegas, J., Alvear, G., \& López, J. (2001) Evaluación y Seguimiento de la Salud de los Trabajadores (PROESSAT), Serie Académicos No. 34, México: División de Ciencias Biológicas y de la Salud, Maestría en Ciencias en Salud de los Trabajadores, UAM-Xochimilco.

Olivares, V. (2017). Laudatio: Dra. Christina Maslach, Comprendiendo el Burnout. Ciencia \& Trabajo, 19(58), 59-63. http://doi.org/10.4067/S071824492017000100059

Organización Internacional del Trabajo. (2016). Estrés en el trabajo: un reto colectivo. (Primera edición). Italia: Centro Internacional de Formación de la OIT. Recuperado de https://www.ilo.org/wcmsp5/ groups/public/---ed_protect/---protrav/---safework/ documents/publication/wcms_466549.pdf

Organización Mundial de la Salud. (2004). La organización del trabajo y el estrés. Serie protección de la salud de los trabajadores $\mathrm{N}^{\circ}$ 3. Ginebra, Suiza: Biblioteca de la OMS. Recuperado de https://www.who.int/ occupational_health/publications/pwh3sp.pdf

Orozco-Alvarado, J., Núñez-Martínez, P., \& Orozco-Bravo, M. (2019). Los retos de la educación superior de México en el siglo XXI. InterSedes, 20(41), 74-84. https://doi. org/10.15517/isucr.v20i41.38807

Patlán, J. (2019). ¿Qué es el estrés laboral y cómo medirlo? Salud Uninorte, 35(1), 156-184. Recuperado de http://www.scielo.org.co/pdf/sun/v35n1/20117531-sun-35-01-156.pdf https://doi.org/10.14482/ sun.35.1.158.72

Pearlin, L. (2010). The life course and the stress process: Some conceptual comparisons. Journal of Gerontology: Social Sciences, 65(2), 207-215. Recuperado de https://academic.oup.com/psychsocgerontology/ article/65B/2/207/640399 https://doi.org/10.1093/ geronb/gbp106

Pérez, J. (2013). Las publicaciones científicas en Colombia, su origen y su futuro según el nuevo modelo de medición de Publindex. Biosalud, 12(2), 5-6. Recuperado de http://www.scielo.org.co/pdf/biosa/ v12n2/v12n2a01.pdf

Pérez, L. A. (2004). Factores psicosociales laborales, estrés e infarto agudo del miocardio en trabajadores colombianos (Tesis de maestría). Universidad de los Andes, Bogotá, Colombia. Recuperado de https://repositorio.uniandes. 
Rojas-Solís, J., Flores-Meza, G. \& Cuaya-Itzcoatl, I.

edu.co/flexpaper/handle/1992/10385/u251059. pdf?sequence $=1$ \&isAllowed $=y \#$ page $=1$

Poltorak, M. (2018). Brecha laboral por motivos de género: diagnóstico y desafíos. Revista Derechos en Acción, 9, 505-524. https://doi.org/10.24215/25251678e238

Ramírez, J. (2019). El estrés laboral desde una perspectiva relacional. Un modelo interpretativo. Revista Colombiana de Antropología, 55(2), 117-147. http:/l doi.org/10.22380/2539472x.802

Ramírez, T. G. (2018). Estrés laboral en auxiliares administrativos y secretarias en una universidad pública del estado de México (Tesis de licenciatura). Universidad Autónoma del Estado de México. Estado de México. Recuperado de http://ri.uaemex.mx/ bitstream/handle/20.500.11799/98618/Tesis\%202805-2018_Tere.pdf?sequence=3\&isAllowed $=y$

Ramos, V., \& Jordão, F. (2015). La relación entre el estrés laboral, las fuentes que dan el origen y las estrategias de afrontamiento en el sector público y privado. Revista de Psicología del Trabajo y de las Organizaciones, 31(1), 11-20. http://doi.org/10.1016/j. rpto.2014.11.008

Razo, A. M., Díaz, R., \& López, M. P. (2018). Construcción de las Representaciones Sociales de la Calidad de Vida en diferentes etapas de la edad adulta. Espacio Abierto 27(1), 149-167. Recuperado de https:// dialnet.unirioja.es/descarga/articulo/6473195.pdf

Rivera, L. (2010). Definiendo el estrés. Los Sindromes de Estres, Primera edición. Madrid: Síntesis. Recuperado de https://www.researchgate.net/ publication/313503877

Rivera-Encinas, M. T. (2016). Efectividad del uso del mate de coca en la reducción del estrés laboral en administrativos de una universidad. Horizonte Médico, 16(3), 20-28. Recuperado de https://www. redalyc.org/pdf/3716/371647508004.pdf https:// doi.org/10.24265/horizmed.2016.v16n3.04

Rodríguez, D. C. (2017). Legislación laboral para el teletrabajo. Revista Principia Iuris, 14(27), 166-183. Recuperado de http://revistas.ustatunja.edu.co/ index.php/piuris/article/view/1406

Rodríguez, R., \& Rivas, S. (2011). Los procesos de estrés laboral y desgaste profesional (burnout): diferenciación, actualización y líneas de intervención. Medicina y seguridad del trabajo, 57(1), 72-78. http://doi. org/10.4321/S0465-546X2011000500006

*Romero, C., Beleño, R., Ucros, M., Echeverría, A., \& Lasprilla, S. (2016). Factores de riesgos psicosociales extralaborales en personal administrativo universitario. Revista Enfermería actual en Costa Rica, (31), 1-15. http://doi.org/10.15517/revenf. v0i31.22083

Sánchez-Contreras, M. F., \& Murga-Menoyo, M. A. (2019). El profesorado universitario ante el proceso de ambientalización curricular. Sensibilidad ambiental y práctica docente innovadora. Revista Mexicana de Investigación Educativa, 82(24), 765787. Recuperado de http://www.scielo.org.mx/pdf/ rmie/v24n82/1405-6666-rmie-24-82-765.pdf

Sánchez, R. (2017). Factores sociolaborales y estrés en docentes de secundaria de la ciudad de Huancayo. (Tesis de Maestría). Universidad Nacional del Centro del Perú.

Siegrist, J. (1996). Adverse Health Effects of High-Effort/ Low-Reward Conditions. Journal of Occupational Health Psychology, 1(1), 27-41. https://doi. org/10.1037/1076-899.1.1.27

Silva-Ramos, M. F., López-Cocotle, J. J., \& Meza-Zamora, M. E. (2020). Estrés académico en estudiantes universitarios. Investigación y Ciencia de la Universidad Autónoma de Aguascalientes, 28(79), 75-83. Recuperado de https://www.redalyc.org/ jatsRepo/674/67462875008/67462875008.pdf

Soto, D. E., \& Forero, A. (2016). La Universidad Latinoamericana y del Caribe en los Desafíos del Siglo XXI. Revista Historia de la Educación Latinoamericana, 18(26), 279-309. http://doi. org/10.19053/01227238.4375

Unda, S., Uribe, F., Jurado, S., García, M., Tovalín, H., \& Juárez, A. (2016). Construcción de una escala para evaluar factores de riesgo psicosocial en el trabajo en profesores. Revista de Psicología del Trabajo y de las Organizaciones, 32(2), 67-74. http://doi. org/10.1016/j.rpto.2016.04.004

Valle, G. (2018). Teletrabajo, necesidad y solución en Méjico. Un estudio exploratorio de sus barreras culturales y obstáculos. Teuken Bidikay, 9(12), 153-176. Recuperado de https://revistas.elpoli.edu.co/index. php/teu/article/download/1327/1075. https://doi. org/10.33571/teuken.v9n12a7

Vicente, M. T., Torres, J. I., Torres, A., Ramírez, M. V., \& Capdevila, L. (2018). El teletrabajo en salud laboral: Aspectos médico-legales y laborales. Revista CES Derecho, 9(2), 287-297. http://doi.org/10.21615/ cesder.9.2.6

Villalobos, G. (2010). Estudio para el diseño de una batería de instrumentos para la evaluación de factores psicosociales. Colombia: Pontificia Universidad 
Javeriana, Ministerio de la Protección Social. Recuperado de https://portal.posipedia.co/wpcontent/uploads/2019/08/bateria-instrumentoevaluacion-factores-riesgo-psicosocial.pdf

Watts, J., \& Robertson, N. (2011). Burnout in university teaching staff: a systematic literature review. Educational Research, 53(1), 33-50. https://doi.org/1 $0.1080 / 00131881.2011 .552235$

Yousefi, M., \& Abdullah, A. G. K. (2019). The Impact of Organizational Stressors on Job Performance among Academic Staff. International Journal of Instruction, 12(3), 561-576. https://doi. $\operatorname{org} / 10.29333 / \mathrm{iji} .2019 .12334 \mathrm{a}$

Zuniga-Jara, S., \& Pizarro-Leon, V. (2018). Mediciones de Estrés Laboral en Docentes de un Colegio Público Regional Chileno. Información Tecnológica, 29(1), 171-180. http://doi.org/10.4067/S0718 07642018000100171

Zúñiga, M., Pérez, C., \& García, M. (2016). Retos de los académicos en la producción de conocimiento en las universidades públicas estatales de México. Revista Electrónica Pesquiseduca, 8(16), 295-315. Recuperado de https://pdfs.semanticscholar.org/1476/5be11b5ade798db15179bd42e6786c307700.pdf

RIDU / Revista Digital de Investigación en Docencia Universitaria / e-ISSN: 2223-2516

(c) Los autores. Este artículo es publicado por la Revista Digital de Investigación en Docencia Universitaria del Área de Institutional Research and Effectiveness de la Dirección de Aseguramiento de la Calidad, Universidad Peruana de Ciencias Aplicadas. Este es un artículo de acceso abierto, distribuido bajo los términos de la LicenciaCreativeCommons Atribución-CompartirIgual 4.0 Internacional.( http://creativecommons.org/licenses/by-sa/4.0/), que permite el uso no comercial, distribución y reproducción en cualquier medio, siempre que la obra original sea debidamente citada. "Este es un artículo de acceso abierto, distribuido bajo los términos de la LicenciaCreativeCommons Atribución 4.0 Internacional (CC BY 4.0) (https://creativecommons.org/licenses/by/4.0/ deed.es), que permite el uso, distribución y reproducción en cualquier medio, siempre que la obra original sea debidamente citada." 